\title{
LES TECHNIQLES \\ DE PRISES QUANTITATIVES DU PLANCTON ET LE MODE DE REPRÉSENTATION DES RÉSULTATS
}

\author{
par B. DUSSART
}

Chef de Travaux à la Station Centrale d'Hydrobiologie appliquée, chargé de la Station de Biologie Lacustre.

Depuis que le plancton est étudié, et cela remonte à 70 ans à peine, les chercheurs ont envisagé son étude quantitative. Celle-ci permet, en effet, dans la mesure où elle est possible, de résoudre un nombre considérable de problèmes d'intérêt général et piscicole tels que le mode de nutrition des poissons, la valeur biogènique du milięu aquatique considéré, sa capacité biogènique, les variations saisonnières de sa réserve nutritive, en un mot, elle est à la base de toute étude piscicole sérieuse du milieu lacustre et d'étang ; je n'envisagerai ici que le premier de ces milieux.

Par étude quantitative, on peut considérer :

Soit l'étude globale du plancton, auquel cas une étude quantitative se résume à une mesure de volume et de poids.

Soit l'étude particulière de chaque espèce, auquel cas une telle étude nécessite un comptage.

Le problème de la récolte quantitative du plancton contenu dans les eaux naturelles, aussi bien marines que continentales d'ailleurs, admet trois solutions :

$\mathbf{I}^{\circ}$ La "PÊCEE " proprement dite du plancton, c'est-ă-dire la filtration, au travers d'un filet, d'une quantité donnée d'eau, et la concentration des organismes dans le filet.

$2^{\circ}$ Le "Pompage " de l'ensemble constitué par l'eau et le plancton qu'elle contient, ce qui nécessite ultérieurement la concentration de ce plancton :

soit par filtration de l'eau recueillie,

soit par centrifugation de cette eau,

soit par sédimentation du plancton préalablement tué à l'aide d'un fixateur ou d'un soporifique. 
$3^{\circ}$ La "Prise d'eav ", prise récoltant, comme dans le cas précédent, eau et plancton, et nécessitant les mèmes processus opératoires ultérieurs.

Considénons séparément ces trois procédés :

$$
\text { I. - LA PR̂CHE. }
$$

C'est Hensen qui, en I 882 , utilisa, à ma connaissance, le premier, le filet pour des pèches quantitatives. Par le procédé le plus simple qui vient à l'esprit, on étudie les couches profondes par différence : on pêche en un point donné, à un moment donné, avec un filet donné, tiré à une vitesse donnée, d'une certaine profondeur à la surface, puis d'une profondeur légèrement supérieure à la surface ; par comparaison des résultats, on déduit aisément la teneur du plancton dans l'infervalle de profondeur considéré. (Exemple : prises de ro à o mètre et de 20 à o mètre, d'oủ l'on déduit la quantité de plancton existant entre zo et ro mètres.)

Plus tard, Apstern eut l'idée d'obstruer l'ouverture du filet au moyen d'un clapet à fermeture automatique par messager ; Auerbacr, lui, étrangile son filet à l'aide d'un filin secondaire ; Futhmann adopte une variante de ces procédés ; mais aucune de ces modifications n'enlevait malheureusement au filet ses causes initiales d'erreur, qui sont nombreuses. YuNG

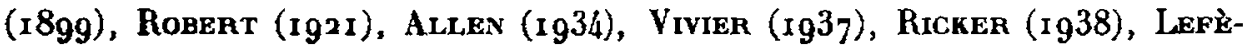
VRE (1946) ont attiré l'attention sur les inconvénients de la méthode du filet, et ont proposé des solutions partielles ou insuffisantes aux problèmes inhérents à cette technique. Je ne m'étendrai donc pas sur ces solutions. Qu'il me suffise de rappeler les principales causes d'incertitude de la techṇique :

- Les mailles du filet ont des dimensions variables avec l'âge du filet et son degré d'humidification (H. Robert, M. LeFìvre) ;

- La quantité de plancton recueillie est fonction de la surface filtrante, donc de la forme du filet (H. RoBers);

- Les organismes colmatent les mailles et réduisent notablement la surface filtrante, cette réduction étant fonction de la quantité du plancton pêchée à un instant donné de la pêche, et de sa composition (M. LEFìvre, W.-E. Ricken);

- L'ouverture du filet joue un rôle primordial dans la capture de certains planctontes bons nageurs (E. Yung, P. Vivier, M. LefèveE) ;

- Le filet ne péche pas pareillement à toutes les vitesses de trainage, d'où la nécessité de tirer le filet toujours à la même vitesse. (C. Apstrin. H. ROBERT).

En ce qui concerne les prises horizontales de plancton, divers appareils ont été proposés dont ceux de Jenkin ( $\mathrm{g}_{9} 3 \mathrm{r}$ ), et de Clancke \& Bumpus (ig39) ainsi que celui de Werner ( 1939 ), pour le plancton sis près du fond. 
Aucun de ces appareils ne permet de véritables prises quantitatives de plancton, d'autant que la répartition de celui-ci est loin d'être uniforme dans l'ensemble d'un lar, d'un étang, ou mème d'une mare. Le filet permet lout au plus de se faire une idée de la composition planctonique d'une masse d'eau, et d'en suivre grossièrement les variations, à condition de ne considérer que quelques espèces ou le résultat global des pèches. Il est. évident que de telles comparaisans ne sont concevables quà condition de procéder aux pêches toujours dans les mèmes conditions, avec les mêmea opérateurs et le mềme appareillage ; la technique du filet ne permet l'étude comparative des masses d'eau entre elles qu'à la seule condition de procéder avec le même engin manié par le même observateur, à toutes les recherches. L'étude comparative des péches planctoniques effectues par des opérateuro différents et au moyen de filets différents n'est possible qu'à condition de standardiser la technique opératoire dans ses moindres détails comme a essayé de le faire ALLEv (Ig34).

En dehors de cette technique de pêche du plancton, il convient de considérer les deux autres méthodes de prises du plancton qui, si elles ne présentent pas les inconvénients de la méthode du filet, en présentent d'autres.

\section{II. - LE POMPAGE.}

Cetfe fechnique déjà vicille (C. Korom la préconise dés la fin du siècle dernier) présente l'avantago de ne dépendre ni de l'opérateur, ni dé la pompe. A. Betayt (rgi 8 et r932), H. Robert (y922), E, Birge \& Ch. Juday (1922), l'ont utilisée avec succès ; mais elle présente trois inconvénients majeurs discutés par ceux même qui ont employế cette méthode, et avec cux II. Lefève: $(19 / 46)$. Ces trois inconvénients sont :

- poids, encombrement, et prix de l'appareil ;

- profondeur limite de prospection réduite $(75$ mètres) ;

- le courant d'aspiration naissant à lextrémité du tuyau provoque un rhêtropisme négatif des espèces naqeant bien et particulièrement des grands Cladocères (Leptodora, Bythotrephes, Daphnia, Sida, Diaphonosoma). Les prises ne donnent donc pas une définition exacte de la composition planetonique en un point donné, mais bien un aperçu relatif. Il existe une conslante de pompe.

Ces divers inconvénients ont empíché l'essor de celte technique couteuse, mais nettement plus précise que celle du filet, bien qu'encore imparfaite.

L'imperfection de la méthode provient également du fait qu'une fois l'eau ainsi pompée, il faut en extraire le plancton. A cette fin, A. Betays (rgi8) utilise un filet à planctón à mailles très fines. E. Birge et Ch. Juday (r922) préfêrent centrifuger l'eau dans une "Crémeuse ". C'est ce qui convient le mieux d'ailleurs, d'après les quelqques auteurs qui ont pu utiliser la technique. 


\section{III. - La phise d'eau.}

C'est certainement la technique idéale pour avoir une représentation réelle de la quantité de plancton contenue dans un certain volume d'eau mais si, théoriquement, cette technique est. parfaite, on se heurte pratiquement à des difficultés énormes. La première est sans conteste la dimension de l'appareil à concevoir.

Pour K. Strøm (1931) el d'après V. Petrensson (1926), soo litres d'ean sont nécessaires pour l'élude du plancton de la Baltique ; d'après les résultats de $A$. Betant $\left(\mathrm{I}^{3}\right)_{2}$ ), il faudrait $x$ mètre cube on au moins 500 litres pour le Léman. Et pour avoir un appareil utilisable et transportable, il ne faut guère dépasser une contenance de 20 litres ! C'est ce quu'ont réalisé C. Koford (19o5), et K. STnøM ( 1931 ) ; la bouteille de roo litres nécessitant un appareillage important a été conçue par J. HJonT et J. Ruud (r927), celle de 200 litres par V. Petrensson (ig26).

Pour l'étude des petites masses d'eau, M. LeF̌̀vne (1946) emploie le moyen simpliste et économique de la bouteille ordinaire qu'on débouche avec une ficelle altachée au bouchon, moyen que E. Navmaxin ( 1939 ) préconise en employant un appareil un peu plus perfectionné.

Ces deux derniers appareils, tout comme celui de C. Koroin, d'ailleurs, présentent le gros inconvénient de perturber notablement le milieu aquatique aux environs de la prise d'eau par suite des remous provoqués par l'échappement de l'air contenu dans la "bouteille ". Cet inconvénient provient de 1'absence de couvercle inféricur.

J. Lund, utilise, pour étudier le phytoplancton du Windermere, un tube, genre tuyau d’arrosage, alourdi à une extrémité, quìl plonge verticalement dans l'eau. Après obturation de I'extrémilé supérieure, il relêve le tube et siphonne son contenù dans un flacon. Il récolte ainsi à peu près quantitativement une colonne d'eau de quelques centimètres cubes de section ca de ro mètres au plus de hauteur.

P. Van Ore ( $9^{3} 7$ ) utilise pour les caux d'étang un seau de so litres qui permet des prises de surface relativement quantitative, cependant imparfait pour le zooplancton.

Un gros progrès a élé réalisé par lutilisation d’appareils du type Petterssox, qui n'est qu'une pompe travaillant sur place, et pouvant recueillir le plancton de 200 litres d'eat, mais il y a cncore de notables causes d'erreur, et l'appareil est difficile à manipuler.

La bouteille de Hort et Ruvb, utilisant le principe de la houteille de Korotn, permet la prise de 100 litres d'eau, cau pénétrant par l'orifice inférieur, les perturbations du miliea à étudier étant minimes relativement (bouteille de type Rutr.ser).

La bouteille de Strøi ressemble à une grande bouteille Friedivger de 20 litres avec deux fenètres de soie filtrante très fine. Cette bouteille 
recueille donc 20 litres d'eau, et le plancton y-inclus se trouve automatiquement concrntré dans a à 2 litres d'eau une fois la prise terminée, et la bouteille hors de l'eau. C'est à mon avis un appareil de ce genre qu'il faudrait envisager à l'occasion d'une standardisation des méthodes limnologiques.

Je signale pour mémoire le "piège "à plancton de Jubar (igr6), d'un maniement trop délicat pour être de quelque utilité, malgré les 45 litres d'eau que cet appareil pouvait recueillir, et son principe particulièrement séduisant (W.-E. Ricker, 1938).

Une fois le plancton recueilli, il faut l'étudier.

Dans l'étude quantitative globale, il n'y a qu'une mesure de volume a effectuer, ou, comme l'ont fait E. Bunge et Ch. Judar (rg22), une mesure de teneur en cendres ou en toute autre substance caractéristique du plaricton sec. Ce volume, exprimé en centimètres cubes ou en millimètres rubes, correspond à la quantité de plancton sédimenté le plus souven durant 24 heures après fixation, contenu dans roo litres, r.ooo litres, ou, pour F. Forel (x 904 ) et M. Le Roux (19\%7), dans y mèlre carré, le filet ramassant le plancton d'une certaine section de la surface du lac, la hauteur étant donnée dans la légende sous forme de mesure de profondeur.

E. Pelloni ( 1936 ), compte le nombre d'individus contenus dans I mètre carré de surface (l'indication des profondeurs étant là encore le complément indispensable aux nombres exposés).

Pour A. Brtast, les variables sont le volume de plancton récolté (en millimètres cubes), et le temps (mois). Il y a donc une courbe par profondeur.

Pour H. IJteamorm, (1925), c'est le nombre d'individus par centimètres cubes qui est important à connaître.

P. Burknolder (r933) utilise un mode de représentation analogue à celui de $H$. Lohmavy dont je parlerai tout à l'heure. Il construit un diagramme très suggestif prenant pour abscisses le nombre de centimètres cubes de plancton par mètre cube d'cau, représenté par un longueur proportionnelle à cette quantité, et pour ordonnées, la profondeur. Il y a un diagramme par prise.

Plus fréquemment. les planctonologistes étudient les espèces, ou les groupes d'espèces. séparément, et pour cela opèrent des comptages. Ceuxci sont effectués soit sur la totalité de la prise, soit sur une portion de cette prise préalablement homogénéisée (par addition de glycérine). Le comptage est effectué, soit dans une cellule de Tromas, soit dans une logette de Kolkwtrz, soit dans une auge de Ulcyotr, soit plus simplement dans une cuve de Dot,russ.

Une fois ces comptages effectués (travail fastidieux), il faut rassembler 
les résultats sous une forme assez pédagogique pour permettre des comparaisons faciles et suggestives. Trois solutions se présentent :

Le tableau.

Le spectre.

Le graphique.

Le tableau est une simple accumulation de documents, suggestive pour. l'auteur, mais le plus souvent peu intéressante pour le lecteur. Il donne soit des nombres absolus, soit encore plus simplement la fréquence deprésence (abondant, rare, etc.).

Le spectre, proposé par P. Van Oxw ( $\mathrm{rg}^{3} 7$ ) est certainement, dans l'état actuel de nos connaissances, la meilleure présentation des résultats. Elle enseigne sur la teneur relative du plancton assez correctement pour permettre des comparaisons sans entrer trop dans le détail, ce qui est inutile tant est peu approfondie notre connaissance de la biocénose planctonique. Il indique suivant un code bien défini, la quantité d'organismes présents dans un certain volume de la prise.

Reste le mode graphique de représentation, c'est de loin le plus courant. Là encore, ce qui rend délical ce mode de représentation, c'est le nombre de variableg :

le temps (durée),

le nombre d'individus,

le volume d'eau considéré,

la profondeur,

le lieu.

P. Bunkholder ( 1933 ) groupe dans le même ğraphique les résultals concernant plusieurs espèces de chaque prise en représentant le nombre de chaque espèce par un grand cercle d'une sphère dont le volume est proportionnel au nombre d'individus de l'spèce considérée par litre d'eau. Les petits cercles sont rassemblés dans le plus grand. Il y a une série de cercles par profondeur (ordonnérs) ct par date (abscisses).

P. Stermann et G. Surbeck (r934), mettent en abscisses le nombre d'individus par litre, représenté par une ligne de longueur proportionnelle à ce nombre, et en ordonnées, la profondleur. Toutes ces lignes ont leur milieu sur la même verticale, et les extrémités de ccs lig̣nes sont réunies à droite. comme à gauche par une lig̣ne brisée. La surface ainsi délimitée donne une idée très suggestive de la variation de l'espèce considérée.

En $1937, P$. Vivien propose une courbe par espèce, le nombre d'ind̆ividus élant porté en abscisses, et la profondeur en ordonnées. Il y a une courbe par prise, donc par date.

Cet auteur a également rassemblé, dans un diagramme un peu touffu, différents résultats, les abscisses représentant le temps, et les ordonnées le nombre d'individus, à une profondeur donnée. Il guit ainsi simultané- 
ment sur le mème graphique l'évolution de plusieurs espèces au cours de l'année.

Il faut remonter à H. Lohiaxix (rgo8) pour a voir une définition du type sphérique de courbes : dans ses diagrammes, tout comme dans ceux de E. Birge et Ch. Jupay (1922), les ordonnées représentent la nombre d'individus, les abcisses, le temps; chez F. Rutrien, les abcisses représentent la profondeur $(937)$. L'unilé de temps est le plus souvent le mois, l'unité d'ordonnées est spéciale, elle mérite attention. Le nombre d'individus est représentẻ par le volume d'une sphère dont la projẹction donne sur le graphique un grand cercle. On ne retient de ce grand cercle que le diamètre parallèle à l'axe des abscisses, diamètre représentant le nombre d'individus.

Cette méthode permet dans une faible surface d'incorporer des écarts de nombres très grands ; c'est son avantage. Elle a cependant le défaut de demander certains calculs qui, bien que facilıtés par des tables, n'en sont pas moins longs et fastidieux. Ces diagrammes ont pourtant une réelle valcur pédagogique. Ils transcrivent en plan des résultats intéressant l'espace (ici un volume d'eau).

$\mathrm{Si}$ je devais formuler une opinion au sujet de celte étude critique des diverses méthodes d'étude quantitative du plancton, et indépendamment de toule préoccupation matérielle ou financière, je proposerais :

La bouteille STrøm ou mieux HJort et Ruun, pour la prise d'eau ;

La centrifugation par centrifugeuse à débit continu (cf. E. Birge et Ch. JUDAY, I 922 ) ;

Le mode de representation par spectre de P. Van Ore ou le mode graphique de H. Lohmaxn ou F. Rutrver suivant la variable envisagée.

Pour une étude globale du plancton, il me semble que la représentation de P. Bupknolden ( 1933 ) est satisfaisante.

\section{LITTERATURE CITÉE}

Allen (W. E.). - Inter. Rev. ges. Hydr. u. Hydrog., 31, pp. 40-65, 1934.

Apstein (C.). - Schr. d. Naturw. Yer. f. Schlesw. Holst., 9, 2.

Betant (A.). - Observations sur le plancton de l'eau du lac de Genève, 1918.

Betant (A.) et Perrienovo (G.). - Études sur la partie occidentale du lac de Genève. Genève, 1932.

Birge (E. A.) et Juday (Ch.). - Wisc. geol. and Nat. Hist. Stirv., 64, Sc. Ser. 13, 1922.

Burkholder (P. R.). - Inter. Rev. ges. Hydrot. n. Hydrog., 28, pp. 202-284, 1933.

Clahke (G. L.) et Bumpes (D. F.). - Inter. Rev. ges. Hytrob. u. Hydrog., 39, pp. 190-192, 1939.

Forel (F. A.). - Le Léman, III, 1904.

Hensen (V.). - Fünf. Ber. Kom. zur wissen. Untersuch. d. deut. Meere, pp. 1-107, 1887.

HJort (J.) et RuUd (J. T.). - Jour. Cons. Int. Expl. Mer, 2, 1, 1927. 
JARnefelt (H.). - Ann. Soc. Zool. Bot. Fenn. Vanamo., 1932-1936.

JENKIN (P. M.). - Inter. Reu. ges. Hydrob. u. Hydrog., 25, pp. 3-45, 1931.

Jyony (Ch.). - Trans. Wisc. Ac. Sc., 18, 2, 1916.

Kofoid (C. A.). - Cons. Int. Expl. Mer, Pub. circ. 32, 1905.

Kolkwirz (R.). - Mitt. aus der K. Prüft. - Anst. f. Wasserversorg., H. 9, Berlin, 1907.

Langeron (M.). - Précis de Microscopie, Paris, 1942.

Lefìvre (M.). - Bull. Fr. Pisc., 142, pp. 21-29, 1946.

LE Roux (M.). - Ann. Biol. lac., 2, pp. 220-387, 1907.

Lohmani (H.). - Jour. N. E. Waterworks Assoc., 14, pp. 1-25, 1899.

Lohmand (H.). - Wissensch. Meersunters. K. Kom., X, p. 192, Kiel, 1908.

Nansen (F.). - Cons. Int. Expl. Mer, Pub. circ. 67, Copenhague, 1915.

Naumann (E.). - Die Binnengewâsser, VI, 1929.

OXE (P. Van). - Inter. Rev. ges. Hydrob. u. Hydrog., 35, pp. 328-338, 1937.

Pelloni (F.). - Contributo all' indagine idrochimica e idrobiologica del Verbano (Bacino di Locarno), p. 1, Locarno, 1936.

Petrenson (V. J.). - Svenska Hydrogr. Biol. Komm. Skr. N. S. Hydrografi, 2, 2, 1926.

Ricker (W. E.). - J. fish. Res. Bd. Can., 4, 1, pp. 19-32, 1938.

Robert (H.). - Bull. Soc. Neuch. Sc. Nat. XLV, 1921.

Robert (H.). - Ann. Biol. lac, XI, p. 208, 1922.

Robext (H.). - Bull. Soc. Neuch. Sc. Nat, XLV, 1921.

Robert (H.). - Ann. Biol. lac, XI, p. 208, 1922.

Rutriner (F.). - Arch. f. Hydrob., 32, pp. 167-319.

Steinmain (P.) et Straeck (G.). - Rev. d'Hydrol., VI, 3/4, pp. 152-215, 1934.

Sтвом (K. M.). - Inter. Rev. ges. Hydrob. u. Hydrog., 25, pp. 266-271, 1931.

Uldyort (P.). - Inter. Rev. ges. Hydrab. u. Hydrog., 34, pp. 15-23, 1927.

Utermonil (H.). - Arch. f. Hydrob., Suppl. Bd. 5, 1925.

UтекмӧнL (H.). - Verh. Int. Ver. I. theor. u. angew. Limnol., V, p. 567, 1931.

Vivier (P.). - Verh. Inter. Ver. f. theor. u. angew. Limnol, VIII, 1, pp. 55-108, 1937.

WeRner (B.). - Intet. Rev. ges. Hydrob. u. Hydrog., 38, pp. 368-371, 1939.

Yung (E.). - Arch. Sc. Phys. et Nat. de Genève, VIII, p. 344, 1899.

Yung (E.). - Arch. Se. Phys. et Nat. de Genève, XIV, 1902. 